\title{
NDE and SHM of Critical Parts using Magnetic and Electromagnetic Methods
}

\author{
M. Witoś ${ }^{a, *}$, M. ZIEJA $^{a}$, N. FAllahi ${ }^{b}$, Z.H. ŻUREK ${ }^{c}$ AND J. KWAŚNIEWski ${ }^{d}$ \\ ${ }^{a}$ Air Force Institute of Technology, Ks. Bolesława 6, 01-494 Warsaw, Poland \\ ${ }^{b}$ I \& T Nardoni Institute, Via della Cascina Pontevica 21, 25124 Brescia, Italy \\ ${ }^{c}$ Silesian University of Technology, Krasińskiego 8, 40-019 Katowice, Poland \\ ${ }^{d}$ AGH University of Science and Technology, al. A. Mickiewicza 30, 30-059 Cracow, Poland
}

\begin{abstract}
The paper has been intended to introduce a complex research problem, that is present in aviation, power engineering, mining and transport, with regard to assurance of operational safety for ageing technology, which is exposed to different form of material degradation. Theoretical reasons of non-destructive evaluation (NDE), structural health monitoring (SHM) and active control of material fatigue have been outlined. The magnetic and electromagnetic methods of NDE and SHM such as metal magnetic memory, low frequency eddy current spectroscopy, Barkhausen noise and 3MA have also been presented. The topic has been illustrated by means of practical examples.
\end{abstract}

DOI: $10.12693 /$ APhysPolA.133.697

PACS/topics: 81.05.Bx, 81.70.Ex, 07.55.-w, 83.85.Lq, 84.37.+q, 75.30Gw, 02.30.Fn, 02.50.Fz

\section{Introduction}

In the operation of machines and equipment there are different material degradation processes, including [1, 2]: surface and intercrystalline corrosion, low-cycle fatigue (LCF), high-cycle fatigue (HCF), very high cycle fatigue (VHCF), thermomechanical cycle fatigue (TMF) and radiation damages which modify material mechanical properties and decrease the ability of the structure to transfer service loads. To reduce the risk of failure the methods of non-destructive evaluation (NDE) and structural health monitoring (SHM) of critical elements are also applied. Among above 150 modern diagnostic methods [3,4], magnetic and electromagnetic nondestructive evaluation methods (MT, EMT) are internationally well recognized.

The article presents selected non-destructive methods at the stage of production, repair and operation of machines and equipment.

\section{Theoretical basics of MT and EMT}

There are some hidden, multi-faceted relations between material type, its technical condition and mechanical as well as physical properties of this material [5]. Thus, it is possible to indirectly diagnose the technical condition of device under test (DUT) by measuring physical properties of the material, what is intentionally used in NDE and SHM methods. In MT and EMT, measurements are taken in a selected frequency band (from range of $\mathrm{DC}$ to the few of $\mathrm{THz}$ ), depending on the applied method [3] and costs of prevention. This test uses different phenomena and physical effects depicted in the literature, i.a.:

*corresponding author; e-mail: witosm@itwl.pl
- internal friction and stress concentration in a surface layer of DUT material [6];

- passage of electric current and eddy currents inducted within the conductor by a variable magnetic field;

- non-linear properties of ferromagnetic materials, i.a.: B-H loop hysteresis, ferromagnetic memory, dependence of magnetic permeability and electrical conductivity on the frequency and microstructure condition [7];

- magneto-mechanical effects (reversible and irreversible) occurring during elastic and plastic deformations of material $[8,9]$;

- broadband magnetic Barkhausen noise generated during low-frequency magnetization of ferromagnetic DUT [10, 11];

- impact of magnetic coupling of coil from DUT on electrical impedance of LC circuit of instrument stalk [12].

\section{Examples}

Applying knowledge from the field of magnetism in technical diagnostics was portrayed on a number of selected NDE and SHM methods.

Metal Magnetic Memory Method (MMM) is a support passive magnetic method of NDE and SHM used to:

- quickly detect cracks,

- conduct a rough quality control of fusion welds,

- remotely detect corrosion in pipelines 
- identify stress concentration zones before open cracks occur

MMM was developed in Russia and China in the 70's of the XX century on the basis of magneto-mechanical effects described in the literature and observation of very high magnetization of defected ferromagnetic elements. Measured and analysed distribution of DC component of magnetic field found in the vicinity of DUT (stray field) - Fig. 1, results from:

- magnetization condition of DUT and local magnetic properties of material (indirectly the technical condition);

- tensor of shape (demagnetization tensor);

- unknown history of DUT operation - valuable diagnostic information recorded in ferromagnetic by mechanical and thermal stresses in the presence of weak external magnetic field $\boldsymbol{H}_{E}$ (Earth's magnetic field, nearby electrical appliances and networks and radio signals)

$$
\boldsymbol{B}_{m}=\boldsymbol{B}_{i}+\boldsymbol{B}_{\text {res }}=\left.\boldsymbol{B}(\boldsymbol{H}, \sigma, T, \% F S, t)\right|_{\boldsymbol{H}_{E}},
$$

where $\boldsymbol{B}_{m}$ - measured magnetic induction in the point with coordinates $(\mathrm{x}, \mathrm{y}, \mathrm{z}) ; \boldsymbol{B}_{i}$ - inductive component of magnetic induction; $\boldsymbol{B}_{\text {res }}$ - residual component of magnetic induction; $\boldsymbol{H}$ - artificial electric field intensity, $\sigma$ - mechanical stress, $T$ - temperature, $\% F S$ - level of structure degradation described by change of: percentage portion of microstructure phases, dislocation density and grains shape, $t$ - time;

- distance of magnetometer from DUT surface.

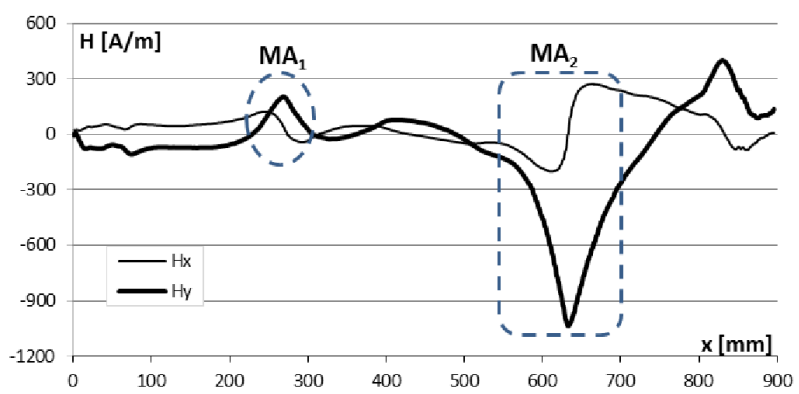

Fig. 1. Magnetogram of ND-37 LP turbine blade after emergency broadband overloads (recording was taken after turbine stoppage and blade was unload, is component tangent to the surface, is component perpendicular to the DUT surface, is magnetic permeability of vacuum, $\mu_{\text {air }} \simeq \mu_{0}$ ).

Under the influence of mechanical and thermal stresses as well as the progressing structure degradation there is a change in distribution trend of magnetic field. There are also magnetic anomalies $\left(M A_{i}\right)$. A reliable interpretation of test results of MMM is connected with obtaining additional information about DUT geometric, distance of magneto-meters from DUT and potential sources of artificial magnetization of DUT material [13]. Quantitative analysis of test results of MMM is controversial due to the lack of reliable data on:

- magnetic properties of construction steels and cast irons, including thermo-mechanical treatment, complex state of stresses and typical degradation processes [14];

- stress inducted magnetization of ferromagnetic in weak magnetic field $[3,9]$.

Increased reliability of diagnosis shall be guaranteed by active magnetic methods e.g. magnetic particle inspection (MPI), rope magnetic testing (RMT), magnetic flux leakage (MFL) [3], due to the fact that DUT fragment (e.g. sheets of transferring pipeline, cables, wire rope) is magnetized with strong magnetic field. During tests Fig. 2a), the following aspects are identified [15]:

- local magnetic anomalies caused by the outflow of magnetic field line (LF) on lateral structure discontinuities (cracks, inclusions) to magnetic field line;

- change of reluctance in magnetic circuit (local changes of magnetic area, LMA) caused by corrosion, material losses or change of DUT geometry.

Demagnetization of DUT is done during production quality control. Active MT utilized in operation of machines alternately with MMM method, makes it difficult to correctly interpret the results of MMM tests in the field of the evaluation of history of maximum stresses, by the simultaneous improvement of dependability of LF and LMA detection in weak magnetic field - Fig. 2b).


Fig. 2. Magnetogram of compacted rope: a) RMT (from the left the signal from internal coil, external coil, halotron and integral from the signal of internal coil, $v_{\text {rope }}=1 \mathrm{~m} / \mathrm{s}$ ); b) MMM (a estimator from array of 16 magnetometers, $\left.v_{\text {rope }}=0.5 \mathrm{~m} / \mathrm{s}\right)$.

Obtaining a reliable diagnostic information on material condition can be done in EMT. In a variable magnetic field $\boldsymbol{H}(t)$ magnetic and electric material prop- 
erties are identified. The widest diagnostic possibilities of steel, cast iron and metal alloys have methods of magnetic Barkhausen noise (MBN), low-frequency spectroscopy of eddy currents (impedance) and 3MA (Micromagnetic Multiparameter Microstructure and Stress Analysis) [3, 9, 16-18].

MBN is a recognized NDE technique for materials characterization of ferromagnetic parts and detection of:

- residual stresses and anisotropy level distributions (monitoring of cold working processes),

- hardness changes,

- grinding burns (different forms of grinding burns can be detected reliably),

- heat treatment defects especially through paramagnetic plate (i.e. landing gears of aircraft, axles, cylinders, bores).

MBN represents a broadband stochastic process (the irreversible jumps of domain wall over pinning sites during the changes in the ferromagnetic magnetization, $\mathrm{f}_{\max }>$ $2 \mathrm{MHz}$ ), therefore the authors, except for the analysis of the envelope of voltage signal (classic approach), apply also stochastic methods - Fig. 3. The noise type is automatically estimated by computing the Power Spectrum Density (PSD) during the preprocess step.
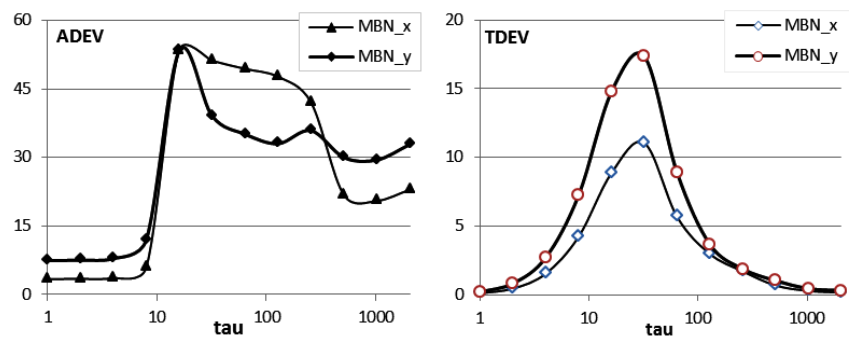

Fig. 3. The overlapping Allan standard deviation (ADEV) and Allan time standard deviation (TDEV) of MBN $\left(|U|=10 \mathrm{~V}, u_{m} \in\langle 0.5 \mathrm{~V} ;-0.5 \mathrm{~V}\rangle, f_{m}=2 \mathrm{~Hz}\right.$, $\left.f_{s}=800 \mathrm{kHz}\right)$.

Eddy currents (EC, also called Foucault currents) are used in NDE and SHM for more than 100 years. To detect structure defects EC are utilized consisting of one or more testing frequencies (a continuous wave method). The use of pulsed eddy currents (PEC) has long been considered for testing metals and it has been applied to operation in specialized areas, such as in the nuclear energy industry. PEC is also applied to diagnose isolated pipelines. Multi-frequency EC and analysis of the level of their harmonics (low-frequency impedance spectroscopy) is used to control the quality of production in car and aviation industry as well as to estimate the material degradation state (during operation and after repair), including $[16,17]$ :

- identification of type and quality of structure (sorting, control of thermo-mechanical treatment),
- parameters of surface layer and diffusion coatings,

- stresses control.

3MA hybrid NDE method, developed at the Fraunhofer Institute for Nondestructive Testing (IZFP), implies a methodical and instrument-specific combination of four different measurement methods:

- multi-frequency eddy current,

- incremental permeability,

- harmonic analysis of the magnetic field

- Barkhausen noise.

After appropriate calibration, the following outcomes can be determined non-destructively: hardness, mechanical para-meters of the tensile test $\left(R_{m}, R_{p 0.2}, R_{e}, \mathrm{~A}_{50}\right.$, etc.), residual stresses, austenite grain size and other. 3MA provides quantitative values of these characteristics, both from the surface and from the interior of the material. Even depth profiles of these characteristics, as well as the hardening depth (case depth, nitriding depth) and the thickness of a ferromagnetic coating can be tested with 3MA. This method is used in industry for many years [18].

\section{Conclusions}

Magnetic and electromagnetic tests are widely applied to control the quality of microstructure and evaluate the technical condition of elements. The quality and reliability of NDE and SHM tests results from:

- proper defining the object under test,

- knowledge of theoretical bases of magnetic effects and material destruction processes,

- dependability of material data bases and numerical models,

- quality of measurement chain and algorithms of measurement signal analysis.

\section{References}

[1] A. Puškár, S.A. Golovin, Fatigue in materials: cumulative damage processes, Elsevier 1985.

[2] H.J. Frost, M.F. Ashby, Deformation-mechanism maps. The Plasticity and creep of Metal and Ceramics, Pergamon Press 1982.

[3] NDTNet database.

[4] Electromagnetic Nondestructive Evaluation (XVII), eds. K. Capova, L. Udpa, L. Janosek, IOP Press 2014.

[5] L. Batista, PhD Thesis, Universität des Saarlandes and Fraunhofer IZFP, Saarbrücken 2014.

[6] M.S. Blanter et al., Internal Friction in Metallic Materials, A Handbook, Springer, Berlin 2007.

[7] J. P. Jakubovics, Magnetism and magnetic materials, 2 Rev. ed., The Institute of Materials, Oxford 1994. 
[8] J. A. Ewing, Magnetic induction in iron and other metals, The Electrician Printing and Publishing Co, London 1900.

[9] Li Lu, Ph.D Thesis, Iowa State University, Ames 2004, paper 1179.

[10] G. Durin, S. Zapperi, in: The Science of Hysteresis: Physical modeling, micromagnetics, and magnetization dynamics, Vol. 2, Ed. I.D. Mayergoyz, Gulf Professional Publishing, 2006, p.181.

[11] J. Pala, J. Bydžovský, Acta Phys. Pol. A 113, 23 (2008).

[12] Keysight Technologies, Impedance Measurement Handbook. A Guide to Measurement Technology and Techniques 6th Ed., Keysight Technologies, 2016.
[13] M. Iwaniec et al., Solid State Phenomena 208, 70 (2014).

[14] MagWeb database.

[15] J. Kwaśniewski, Magnetic testing of wire rope: MTR personnel certification process, AGH, Kraków 2010.

[16] ibg NDT Systems Corporation.

[17] Z.H. Zurek, P. Duka, Circuits for Material Testing and NDT, KOMEL 2015.

[18] G. Dobmann, in: Nuclear Power-Control, Reliability and Human Factor, Ed. P. Tsvetkov, INTECH, Rijeka 2011, p. 311. 